\title{
Detection of Laminariaceae Species Based on PCR by Family-specific ITS Primers
}

\author{
Chang Geun Choi ${ }^{1}$ and Jong-Myoung Kim ${ }^{2 *}$ \\ ${ }^{1}$ Department of Ecological Engineering, ${ }^{2}$ Department of Marine Bio-Materials and Aquaculture, Pukyong National University, \\ Busan 608-737, Korea
}

\begin{abstract}
To analyze nucleotide sequence encoding internal transcribed spacer (ITS) regions specific to the Laminariaceae family, genomic DNA was isolated from six brown algae species distributed along the east coast of Korea. These included three species from the Laminariaceae family (Agarum clathratum Dumortier, Costaria costata [C. Agardh] Saunders, and Saccharina japonica Areschoug) and two species from the Alariaceae family (Undaria pinnatifida [Harvey] Suringer and Ecklonia cava Kjellman), both in the order Laminariales, and one species from the family Sargassaceae in the order Fucales (Sargassum serratifolium). Based on a sequence analysis of ITS-1 and ITS-2 for A. clathratum, C. costata, and E. cava, oligonucleotides were designed from the regions that showed sequence conservation in Laminariaceae. Following polymerase chain reaction using three sets of primers, amplification of ITS-1 and ITS-2 was detected in reactions using genomic DNA isolated from the species belonging to Laminariaceae, but not from the species belonging to the other families. The results indicate that this method can be used for the detection and identification of Laminariaceae species.
\end{abstract}

Key words: ITS-1, ITS-2, Laminariaceae, Species detection

\section{Introduction}

Algae are photosynthetic organisms found in aquatic environments, and they can be divided into three divisions based on pigment content: Chlorophyta, Pheophyta, and Rhodophyta. The brown algae (Phaeophyta) are a group of mostly marine species found in temperate and colder Northern hemisphere waters. Species belonging to 12 orders in the class Phaeophyceae (Ectocarpales, Ralfsiales, Sphacelariales, Dictyotales, Chordariales, Dictyosiphonales, Scytosiphonales, Cutleriales, Sporochnales, Desmarestiales, Laminariales, and Fucales) are known to be distributed along the coast of Korea (Lee and Kang, 2002). Species belonging to three families (Alariaceae, Chordaceae, and Laminariaceae) in the order Laminariales were found in Korea. The former includes Undaria pinnatifida, the most widely cultivated algae in Korea, as well as Ecklonia cava, Ecklonia stolonifera, and Eisenia bicyclis. The
Laminariaceae family is distributed along the east coast of Korea and includes Saccharina japonica, the second most widely cultivated algae in Korea, along with Agarum clathratum and Costaria costata.

Commercial production of kelp species has been mostly achieved by mariculture in East Asian countries including China (the largest producer of Laminaria), Korea, and Japan. Korea is the sixth largest macroalgae producer in the world, producing 921,024 MT in 2008, despite the fact that algal species produced in large amounts are mostly cold currentadapted species, meaning that production is restricted during the winter season. For mass production, it is of commercial interest to screen Laminariaceae species in which most of fastgrowing algal species belong.

Genes encoding ribosomal RNAs and internal transcribed
Open Access http://dx.doi.org/10.5657/FAS.2012.0157

This is an Open Access article distributed under the terms of the Creative Commons Attribution Non-Commercial License (http://creativecommons. org/licenses/by-nc/3.0/) which permits unrestricted non-commercial use, distribution, and reproduction in any medium, provided the original work is properly cited. pISSN: 2234-1749 eISSN: 2234-1757
Received 28 February 2012; Revised 17 May 2012

Accepted 24 May 2012

*Corresponding Author

E-mail: jongkim@pknu.ac.kr 


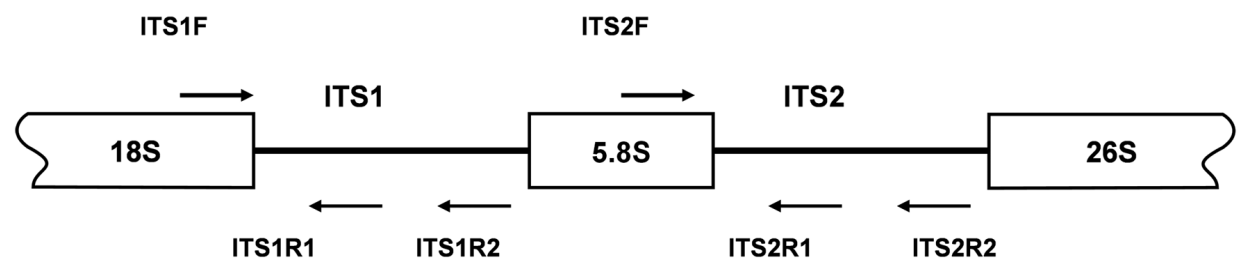

Fig. 1. Diagram showing the locations of oligonucleotides used for the amplification of internal transcribed spacer (ITS)-1 and ITS-2. Figures are not in scale.

spacer (ITS) regions have been used as molecular markers for species identification and phylogenetic analysis of macroalgae (Bird et al., 1992; Ragan et al., 1994). While a rather conserved 18S rRNA sequence provides information for distinguishing evolutionary distant species, a rapidly evolving ITS sequence has been used for the identification of intraspecific variation. In this study, DNA regions encoding ITS-1 and ITS2 of species belonging to Laminariaceae were analyzed to develop a PCR-based species identification method.

\section{Materials and Methods}

\section{Materials}

Macroalgal species including U. pinnatifida, E. cava, A. clathratum, C. costata, S. japonica, and S. serratifolium were collected from the east coast area of Korea. Species collected from at least two different locations were stored at $-20^{\circ} \mathrm{C}$ until DNA isolation was performed. Kits used for plasmid and PCR product purification were purchased from NucleoGen (Seoul, Korea) and pTOP TA V2 cloning vector was obtained from Enzynomics (Daejeon, Korea). Oligonucleotides and a HiQPCR mix were obtained from Genotech (Daejeon, Korea). Oligonucleotides used for the initial amplification of ITS-1 and ITS-2 (Fig. 1) and inner regions were designed as described previously (Yotsukura et al., 1999; Kim and Choi, 2010). Oligonucleotides specific to Laminariaceae ITS-1 and ITS-2 were designed from the corresponding regions conserved in $A$. clathratum and C. costata but not in E. cava (Fig. 2) as follows: 5' - T C C G TAGG T GA A C C T GCGG-3' (I T S 1 F), 5' - GCT GCG T T C T T CATCGATGC-3' (I T S 1 R), 5' - CCAACT TCGCATAACGAATTC (ITS1R1)-3', 5' - AGGTTGGGGGGGCCGCGGC-3' (ITS1R2), 5' - TCCTGGGA GCATGCT TGTCG-3' (ITS2 F), $5^{\prime}$ - T C C T C C GC T TAT T GATAT GC - 3' ( I T S 2 R), 5' -CGAGAGTCGCCGCCGAAGCG-3' (ITS2R1), and 5' -ACGAAAGTGGTACGGTTTCC-3' (ITS2R2).

\section{Isolation of genomic DNA}

Genomic DNA was isolated using previously described nuclei isolation/centyltrimethylammonium bromide (CTAB) methods (Varela-Álvarez et al., 2006). Tissue samples (0.5 g) were ground in a mortar in the presence of liquid nitrogen and homogenized in $5 \mathrm{~mL}$ STE buffer (400 mM sucrose, $50 \mathrm{mM}$ Tris- $\mathrm{Cl} \mathrm{pH} \mathrm{7.8,} 20 \mathrm{mM}$ ethylenediaminetetraacetic acid (EDTA), $0.2 \%$ bovine serum albumin, and $0.2 \%$ $\beta$-mercaptoethanol). The homogenate was filtered through a $50 \mu \mathrm{m}$ nylon mesh by squeezing and was subjected to centrifugation at $1,000 \mathrm{~g}$ for $20 \mathrm{~min}$. The nuclei pellet was resuspended in $50 \mu \mathrm{L}$ CTAB buffer $(2 \%$ CTAB, $2 \%$ polyvinylpyrrolidone, $100 \mathrm{mM}$ Tris- $\mathrm{Cl} \mathrm{pH} 8.0,20 \mathrm{mM}$ EDTA, and $1.4 \mathrm{M} \mathrm{NaCl}$ ) followed by incubation at $65^{\circ} \mathrm{C}$ for $1 \mathrm{~h}$. The suspension was extracted with the same volume of the mixture containing chloroform:isoamylalcohol (24:1) followed by centrifugation at 14,000 rpm for $3 \mathrm{~min}$. DNA in the supernatant was precipitated by the addition of two volumes of ethanol and 0.1 volume of $3 \mathrm{M}$ sodium acetate $(\mathrm{pH}$ 5.2) followed by centrifugation.

\section{Cloning of the genes encoding ITS-1}

PCR amplifications of the regions corresponding to the inner segments of ITS-1 and ITS-2 were carried out with sets of primers including ITS1F together with the species-specific primers ITS1R1 or ITS1R2, and ITS2F together with ITS2R1 or ITS2R2, respectively. Amplification of ITS-1 was carried out in a $20 \mu \mathrm{L}$ PCR mixture including a HiQ-PCR Mix, genomic DNA $(0.1 \mu \mathrm{g})$, and $1 \mu \mathrm{M}$ primers. The reaction was carried out with an initial denaturation at $95^{\circ} \mathrm{C}$ for 5 min followed by 30 cycles consisting of denaturation at $94^{\circ} \mathrm{C}$ for $30 \mathrm{~s}$, annealing at $60^{\circ} \mathrm{C}$ for $30 \mathrm{~s}$, and extension at $72^{\circ} \mathrm{C}$ for $30 \mathrm{~s}$, together with a final extension at $72^{\circ} \mathrm{C}$ for 5 min. PCR amplification using ITS2F and ITS2R1 primers was also carried out under the same condition except an annealing temperature at $65^{\circ} \mathrm{C}$. Amplified PCR products, resolved upon agarose gel electrophoresis, were purified by gel extraction, cloned into the pTOP TA V2 cloning vector, and then transformed into E. coli $\mathrm{DH} 5 \alpha$ as described by Sambrook and Russell (2001). Recombinant plasmids isolated by alkaline lysis were analyzed by EcoRI restriction digestion and DNA sequencing.

\section{Sequence analysis}

The nucleotide sequences obtained were aligned with other ITS sequences using ClustalW (Thompson et al., 1994). The 
A

AgarcITS1

CostcITs1 EckcITS1

AgarcITS1 CostcITS1 EckcITS1

AgarcITS1 CostcITs1 EckcITS1

AgarcITS1 CostcITS1 EckcITS1

AgarcITS1 CostcITs1 EckcITS1

B

AgarcITS2 CostcITs2 EckcITS2

AgarcITS2 CostcITs2 EckcITS2

AgarcITS2 CostcITS2 EckcITS2

AgarcITS2 CostcITS2 EckcITS2

AgarcITS2 CostcITS2 EckcITS2

\section{ITS1F}

TCCGTAGGTGAACCTGCGGAAGGATCATTACCGAACAGCGGGAGGTTTCATATACCC--GCTTTATAAATTGTCTCGGCC 78 TCCGTAGGTGAACCTGCGGAAGGATCATTACCGAACAGCGGGAGGTTTCATATACCC--GCTCTATAAATTGTCTCGGCC 78 TCCGTAGGTGAACCTGCGGAAGGATCATTACCGAA-AGCGGGTTCGTTCACACCCCCCCGCTCTATAAATTGTCTGAGAC 79

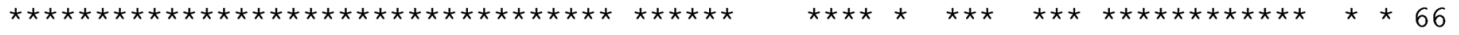

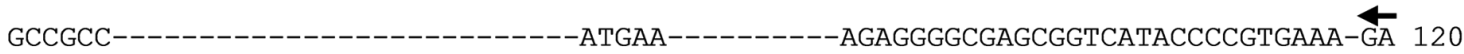
ACCGCC------------------------ATGAA---------AGAGGGGCGAGTGGTCATACCCCGTGAAACGA 121 GTCGCCGTTTGTAACCTCATCTTTTTTAATTTAATTAATTAAAACCCGAGGGAGGCGAGCGTTTATACCCCGAGAAA-GA 158

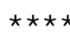

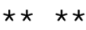

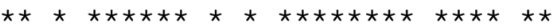

\section{ITS1R1}

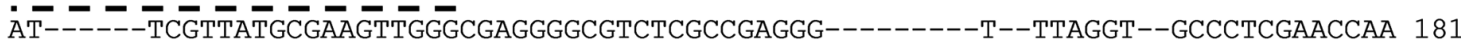
AT------TCGTTATGCGAAGTTGGGCGAGGGGCGTCTCGCCGAGGG---------TCTTTCGCTAGGCTCTCGAACCAA 186 TTCTTTTTTTGTTATGCGAAGTTGGGCGAGGGGCGCCTCGCCGCGAGAGCGAGTTTTTTCTCGCT---CTCTCGAACCAA 235

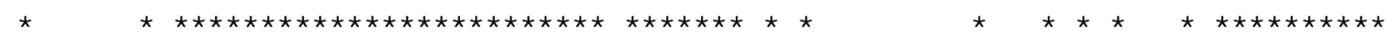

AGCGCACCCCACAT-TTCAACCCC-ATTAAACTCTGAATCTGAACTCAAAGGGGGGCAG-----CGCTTG---CCGCGG 251 AGCGCACCCCACACATTCAACCCC-ACTAAACTCTGAATCTGAACTCAAAGGGAGGCAGGCGG-CGCTCGTCGCCGCGGC 264 AGCGCACCCCACACAATCAACCCCGATGAAACTCTGAATCTGAACTCAAAGGGGGGCCCTCCCTCACACGGGGGCGGGCT 315

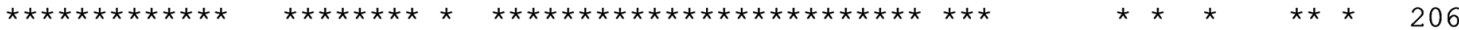

$\overline{\mathrm{C}} \overline{\mathrm{CC}} \overline{\mathrm{CC}} \overline{\mathrm{CA}} \overline{\mathrm{AC}} \overline{\mathrm{C}}-\mathrm{TT} T \overline{\mathrm{A}} \mathrm{ACGTTGTAAAACTTTCAGCGACGGATGTCTTGGCTCCCGCATCGATGAAGAACGCAGC} 323$ CCCCCCAACC-TTTAACGTTGTAAAACTTTCAGCGACGGATGTCTTGGCTCCCGCATCGATGAAGAACGCAGC 336 CCCCCCAACCATTTAACGTTGTAAAACTTTCAGCGACGGATGTCTTGGCTCCCGCATCGATGAAGAACGCAGC 388

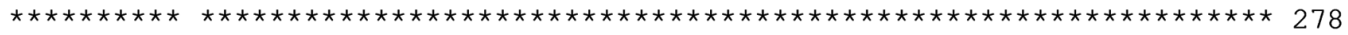

ITS2F

TCCTGGGAGCATGCTTGTCGGAGTGTCTGTTGACACCACTCGCCCCCC-----TTCT------CTCCC---CCCTGTAAC 66 TCCTGGGAGCATGCTTGTCGGAGTGTCTGTTGACACCACTCGCCCCСCСTCTCTTCT------CTCCCGTTCCCCGTAAC 74 TCCTGGGAGCATGCTTGTCGGAGTGT-TGTTGACACCACTCGCCCCCGTCTTCTTCTTCTTGGCTCTTCTCCCCTCTAAT 79

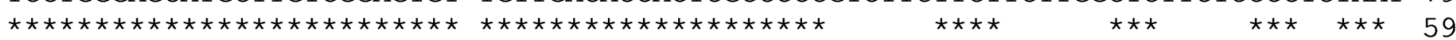
---------------AGGGG-----CGGGGGC----GATCG---------CGGGGCGGACTCTGAGTGTTCCGGAGTT 111 -AAGGGTCGTGCGTGGGCAAGAGATCG--------CGGGGCGGACTCTGAGTGTTCCGGAGCT 128 CCCGACTTTCTTTGAAAGGGGGGCGGGGGGGGGGGAGGGGTCGGATTTTGAACGGGGCGGACTCTGAGTGTTCCGGAGTT 159

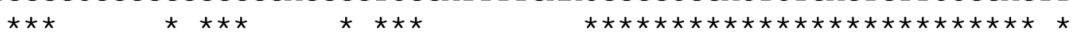

--CCCATGCTCCGAGTGCACCTAATCTCGTGAACGAAGCCTCTCGCGCCCTGCCGCACA-GAGTTGTTGACGGCGCT--- 185 TACCCATGCTCCGAGTGCACCTAATCTCGTGAACGAAGCCTCTCGCGCCCTGCCGCACA-GAGTTGTTGACGGCGCT--- 204 T------CTCCGAGTGCACCTAATCTCGTGAAGGAAGCCTCTCGCGCCCTGCCGCACAAGAGTTGTTGACGGCGCTTTG 232

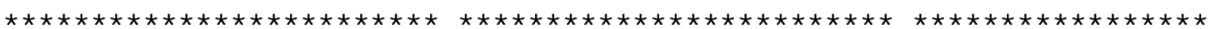

ITS22R1

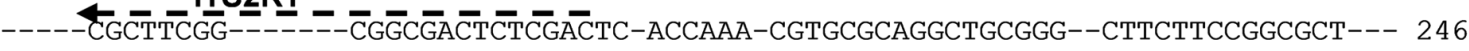
-----CGCTTCGG------_CGGCGACTCTCGGCTC-ACCAAA-CGTGCGCAGGATGCGGGGCCTCATTCCGGCGCT--- 267 TTTGCCCCTCCGGGGCGGGCCGGCGACTCTCGACTCTGCCAAAACGTGCGCAGGCTGCGGGG-CTTCTTCCGGCGCGGCG 311

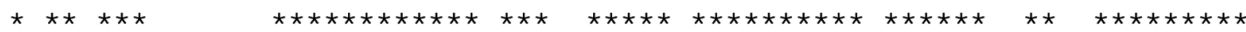
ITS2R2

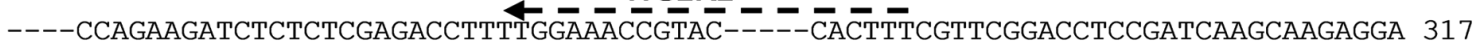
----CCAGAAC-TTTTTATTGTGA-----TGGAAACCGTAC-----CACTTTCGTTCGGACCTCCGATCAAGCAAGAGGA 332 GCGGCCCGAACGTTTCTTTTGTTCG-TTCGGGATGCTATGCATCCACACTTTCGTTCGGACCTCCGATCAAGCAAGAGGA 390

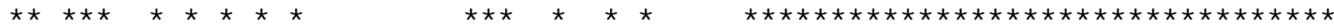

Fig. 2. Comparison of internal transcribed spacer (ITS)-1 (A) and ITS-2 (B) sequences of Agarum clathratum (AgarclTS1 and AgarclTS2), Costaria costata (CostcITS1 and CostcITS2), and Ecklonia cava (EckclTS1 and EckcITS2) using Clustal W. Nucleotide sequences at the ends were trimmed to make the same length for comparison and the broken arrows indicates the positions of ITS-specific primers used for PCR amplification.

accession numbers of ITS-1and ITS-2 sequences, acquired from the NCBI GenBank database (Guiry and Guiry, 2011) were as follows: E. cava Kjellman (AF319009), A. clathratum Dumortier (FJ042768), and C. costata Saunders (AF319027).

\section{Results and Discussion}

The production of macroalgae has been increasing in recent years as its demand for commercial application has increased. 


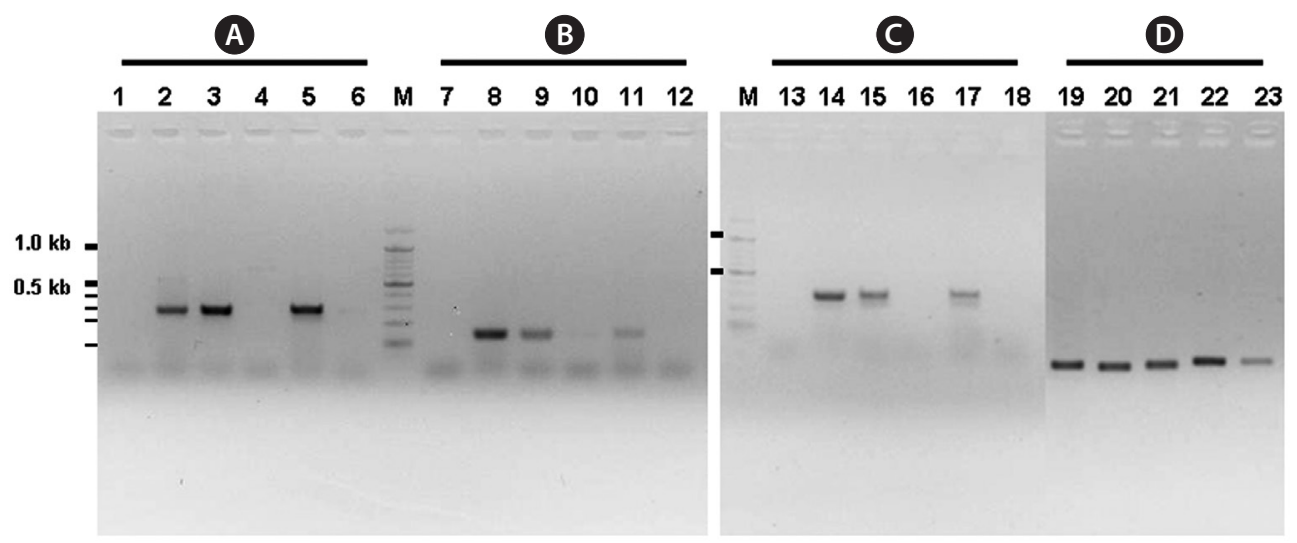

Fig. 3. PCR amplification of the regions encoding internal transcribed spacer (ITS)-1 and ITS-2. PCR was carried out with sets of oligonucleotdes ITS1F together with ITS1R2 (A) or ITS1R1 (B), and ITS2F together with ITS2R2 (C), or ITS2R1 (D). Each lane includes PCR products amplified by using genomic DNA isolated from Ecklonia cava (1, 7, and 13), Agarum clathratum (2, 8, 14, and 19), Saccharina japonica (3, 9, 15, and 20), Undaria pinnatifida (4, 10, 16, and 21), Costaria costata (lanes 5, 11, 17, and 22), and Sargassum serratifolium (lanes $6,12,18$, and 23). PCR were carried out with annealing temperature at $60^{\circ} \mathrm{C}$ (a-c) and $65^{\circ} \mathrm{C}(\mathrm{d})$ and products were analyzed by $1.5 \%$ agarose gel electrophoresis. Lanes $\mathrm{M}$ include $100 \mathrm{bp}$ size markers and thick bars indicate 1.0 and $0.5 \mathrm{~kb}$, respectively.

In Korea, the production of major algal species including $U n$ daria, Saccharina, and Porphyra becomes restricted in the winter season. To extend the seaweed cultivation industry to meet an increasing demand, it is necessary to find a species that can be produced throughout the year or that can grow during the high temperature season with a fast rate of productivity. Species belonging to Laminariaceae are candidates for this screening as the family includes species such as Macrocystis, the largest known seaweed, and Saccharina, which is produced in large amounts in Korea.

Algal species are classified by phenotypic characteristics as well as genetic markers (Yoon et al. 2001; Saunders, 2005). Differences in the morphological characteristics including the presence of mucilaginous organs in the sporophyte, lack of an eyespot in meiospores, and uniflagellation of the sperm exist among the families in the order Laminariales (Kawai and Sasaki, 2000). The Laminariaceae family is characterized by the presence of a distinct stipe, paraphyses without hyaline appendages, and the absence of outgrowths or splitting of the transition zone. In particular, S. japonica has a single, cylindrical stipe and a holdfast with multibranched haptera. The thallus of $C$. costata has a branched holdfast, a flattened and finely grooved stipe, and an elliptical blade up to $2 \mathrm{~m}$ long and $30 \mathrm{~cm}$ wide, with parallel ribs running along its length. The thallus of A. clathratum has a branched holdfast, a stipe up to $30 \mathrm{~cm}$ long, a stiff blade riddled with small but distinctive holes, and a wide midrib. To perform a detailed classification of Laminariaceae species and to develop a genetic markerbased detection method, nucleotide sequence-encoding ITS regions were analyzed for six brown algae species collected from coastal areas of Korea. These included five species from the order Laminariales and one species from the order Fucales. The former included three species of Laminariaceae $(A$. clathratum, C. costata, and S. japonica) and two species of Alariaceae (U. pinnatifida and E. cava). A relatively distant member belonging to the family Sargassaceae of the order Fucales, Sargassum serratifolium, was also included.

The isolation of genomic DNA from macroalgae and its subsequent use in PCR are known to be hindered by polysaccharides and phenolic compounds (Koonjul et al., 1999; Varma et al., 2007). Among the methods used for genomic DNA isolation from macroalgae (Varela-Álvarez et al., 2006; Hoarau et al., 2007; Snirc et al., 2010), the STE and CTAB buffer method was used for the species tested in this study. Upon confirming the integrity of genomic DNA by agarose gel electrophoresis (data not shown), DNA was used as a template for amplification of the regions corresponding to ITS. The typical ribosome in algae is composed of four rRNAs of which the rRNA cluster of $18 \mathrm{~S}-5.8 \mathrm{~S}-26 \mathrm{~S}$ is transcribed from a single transcriptional unit (Coleman and Mai, 1997; Yotsukura et al., 1999; Torres-Machorro et al, 2010). In this study, PCR amplification of regions encoding ITS-1 and ITS-2 using a genomic DNA template and primers (ITS1F/ITS1R and ITS2F/ ITS2R) resulted in 250-300 bp ITS-1 and ITS-2 fragments depending on the species, similar to the sizes reported from other macroalgal species (data not shown). At least three independent clones were analyzed to confirm the ITS sequences presented as many copies in the genome. A sequence comparison of ITS-1 and ITS-2 was first performed with three closely related species, C. costata, A. clathratum, and E. cava, to detect the regions that have differentiated between the species (Fig. 2). The former two, A. clathratum and C. costata belonging to the family Laminariaceae, and E. cava belonging to the neighboring family Alariaceae, were barely distinguishable, with almost identical $18 \mathrm{~s}$ rRNAs except for a single divergence at one position (data not shown). The sequence analy- 
sis of DNA fragments encoding the ITS-1 region obtained in this study showed $100 \%$ identity with the sequence reported from C. costata (AF319027) and A. clathratum (FJ042768), and $97 \%$ identity with the sequence reported from $E$. cava (AF3190009). Analysis of ITS-2 sequences also showed complete identity with those reported from the corresponding species, C. costata (AF319027), A. clathratum (FJ042768), and E. cava (AF319009). Sequence comparison among the species indicated that $A$. clathratum and C. costata, showing $91 \%$ and $89 \%$ identity with ITS- 1 and ITS-2, respectively, are more conserved when compared to E. cava. Based on this result, Laminariaceae-specific oligonucleotides were designed from the regions conserved in A. clathratum and C. costata but not in E. cava (Fig. 2).

The inner segments of ITS-1 and ITS-2 were amplified using primers specific to the Laminariaceae family designed in this study. In PCRs using three sets of primers, amplified products were detected in reactions using genomic DNA isolated from A. clathratum, $S$. japonica, and C. costata, all of which are members of Laminariaceae (Fig. 3A-3C). The sizes of the PCR products were 262 bp, 139 bp, and 292 bp, respectively, as expected from the sequences. No amplified products were observed in reactions using genomic DNA templates isolated from $U$. pinnatifida and E. cava belonging to Alariaceae, and the relatively distantly related species Sargassum serratifolum. This suggests Laminariaceae-specific ITS-1 and ITS-2 amplification only in a PCR using three sets of ITS primers including ITS1R1, ITS1R2, and ITS2R2. For PCR amplification with ITS2F and ITS2R1, amplified products of $200 \mathrm{bp}$ were observed in all samples under the same reaction conditions (data not shown). PCR amplification was also carried out at an annealing temperature of $65^{\circ} \mathrm{C}$ to exclude the possibility of nonspecific amplification due to the lower annealing condition $\left(60^{\circ} \mathrm{C}\right)$ used above. The result also showed amplification of the fragments in all samples under the same reaction conditions although the intensity of the fragment in Sargassum was slightly lower than in the others tested (Fig. 3D). This suggests that the region corresponding to the ITS2R1 primer is more conserved in brown algae than the other ITS regions. Therefore, the ITS2R1 primer can be used for ITS-2 amplification in most of Phaeophyceae species but may not be a good primer for specific detection of Laminariaceae species.

The present study clearly showed that only genomic DNA isolated from the family Laminariaceae could be amplified by PCR, indicating that PCR using the primer sets of ITS1F together with ITS1R1 or ITS1R2, and ITS2F together with ITS2R1, can be used for the specific detection of Laminariaceae. This principle can be applied to develop other familyspecific detection methods.

\section{Acknowledgments}

We would like to thank Mr. In-Chul Hwang for technical assistance. This research was supported by a grant from Development of Marine-Bioenergy Program Funded by Ministry of Land, Transport and Maritime Affairs of Korean Government.

\section{References}

Bird CJ, Murphy CA, Rice EL and Ragan MA. 1992. The 18S rRNA gene sequences of four commercially important seaweeds. J Appl Phycol 4, 379-384.

Coleman AW and Mai JC. 1997. Ribosomal DNA ITS-1 and ITS-2 sequence comparison as a tool for predicting genetic relatedness. J Mol Evol 45, 168-177.

Guiry MD and Guiry GM. 2011. Algaebase, World-wide electronic publication [Internet]. National University of Ireland, Galway, IE, Accessed 28 Jun 2011, http://www.algaebase.org.

Hoarau G, Coyer JA, Stam WT and Olsen JL. 2007. A fast and inexpensive DNA extraction/purification protocol for brown macroalgae. Mol Ecol Notes 7, 191-193.

Kawai H and Sasaki H. 2000. Molecular phylogey of the brown algal genera Akkesiphycus and Halosiphon (Laminariales), resulting in the circumscription of the new families Akkesiphycaceae and Halosiphonaceae. Phycologia 39, 416-428.

Kim JM and Choi CG. 2010. Phylogenetic analysis of Phyllospadix iwatensis based on nucleotide sequences encoding 18S rRNA and ITS-1. Fish Aquat Sci 13, 272-277.

Koonjul PK, Brandt WF, Farrant JM and Lindsey GG. 1999. Inclusion of polyvinylpyrrolidone in the polymerase chain reaction reverses the inhibitory effects of polyphenolic contamination of RNA. Nucleic Acids Res 27, 915-916.

Lee YP and Kang SY. 2002. A Catalogue of the Seaweeds in Korea. Cheju National University Press, Jeju, KR.

Ragan MA, Bird CJ, Rice EL, Gutell RR, Murphy CA and Singh RK. 1994. A molecular phylogeny of the marine red algae (Rhodophyta) based on the nuclear small-subunit rRNA gene. Proc Natl Acad Sci U S A 91, 7276-7280.

Sambrook J and Russell DW. 2001. Molecular Cloning: A Laboratory Manual. 3rd ed. Cold Spring Harbor Laboratory Press, Plainview, NY, US.

Saunders GW. 2005. Applying DNA barcoding to red macroalgae: a preliminary appraisal holds promise for future applications. Philos Trans R Soc Lond B Biol Sci 360, 1879-1888.

Snirc A, Silberfeld T, Bonnet J, Tillier A, Tuffet S and Sun JS. 2010. Optimization of DNA extraction from brown algae (Phaeophyceae) based on a commercial kit. J Phycol 46, 616-621.

Thompson JD, Higgins DG and Gibson TJ. 1994. CLUSTAL W: improving the sensitivity of progressive multiple sequence alignment through sequence weighting, position-specific gap penalties and weight matrix choice. Nucleic Acids Res 22, 4673-4680.

Torres-Machorro AL, Hernández R, Cevallos AM and Lópex-Villaseñor I. 2010. Ribosomal RNA genes in eukaryotic microorganisms: witnesses of phylogeny? FEMS Microbiol Rev 34, 59-86.

Varela-Álvarez E, Andreakis N, Lago-Lestón A, Pearson GA, Serrão 
EA, Procaccini G, Duarte CM and Marbá N. 2006. Genomic DNA isolation from green and brown algae (Caulerpales and Fucales) for microsatellite library construction. J Phycol 42, 741-745.

Varma A, Padh H and Shrivastava N. 2007. Plant genomic DNA isolation: an art or a science. Biotechnol J 2, 386-392.

Yoon HS, Lee JY, Boo SM and Bhattacharya D. 2001. Phylogeny of Alariaceae, Laminariaceae, and Lessoniaceae (Phaeophyceae) based on plastid-encoded Rubisco spacer and nuclear-encoded ITS sequence comparisons. Mol Phylogenet Evol 21, 231-243.

Yotsukura N, Denboh T, Motomura T, Horiguchi T, Coleman AW and Ichimura T. 1999. Little divergence in ribosomal DNA internal transcribed spacer-1 and -2 sequences among non-digitate species of Laminaria (Phaeophyceae) from Hokkaido, Japan. Phycol Res $47,71-80$. 